\title{
Explaining the Earth System -- Based on the Theory of Earth Expansion
}

\author{
Subhasis Sen \\ Retired Scientist, (Council of Scientific and Industrial Research, India), O-26, Patuli, Kolkata, 700094, India \\ ssennagpur82@yahoo.com
}

\begin{abstract}
Here following the Earth expansion theory, a new interpretation on Earth has been put forward, bearing in mind that the original Earth was small, ocean-less and covered with a solid sialic Crust. Geophysical studies have confirmed that below the Crust there occurs a basaltic Mantle, followed by fluid Outer Core - considered to be composed of liquid iron. The planet's inner-most part or Inner Core, though composed of solid iron from where magnetic lines of force emerge, has not been reckoned as magnetic because of high core temperature. Here we have shown that since the thickness of the Outer Core matches with the extent of expansion, the former has opened up as a void zone due to expansion. Hence, in the planet's interior two solid geospheres separated by a void zone occur which in turn would give rise to a force of gravitation in the reverse direction. We interpret here that because of reverse gravity, temperature of the solid Inner Core would be sufficiently low and the Inner Core, therefore, is a magnet. This view explains magnetic features of the planet, including pole reversal and polar wandering. The concept additionally explains origin of oceans, continental drift, nature of terrestrial geospheres, etc.
\end{abstract}

Keywords: Earth Expansion Theory, Oceans, Mantle. Void Geosphere, Inner Core, Magnetism, Reverse Gravity

\section{Introduction}

Ever since the first authentic atlas of the world was prepared in 1570 by Abraham Ortilius ${ }^{1}$, the parallel shore lines of Africa and South America became conspicuous. The pioneer cartographer not only noted this remarkable feature, but also for the first-time proposed occurrence of continents in a conjoined manner in the past. After that several observers contributed on the possibility of occurrence of continents conjointly in the past. amongst whom the work of Alfred Wegener ${ }^{2}$, a meteorologist from Germany, presented in 1912 attracted much attention. The fitting of continents proposed earlier by Antonio Snider-Pellegrini ${ }^{3}$ of France as well as that of Wegener could only be achieved by liberally distorting the boundary of the relevant continents. Hence these works, despite their historical importance, should not be considered as sacrosanct.

Nevertheless, considering mainly the rigid condition of the mantle, coupled with improper adjustment of land masses, the view of continental drift was criticized by many, especially by the renowned British scientist Harold Jeffreys ${ }^{4}$ in 1926 and the concept was eventually rejected. After several decades the concept of continental drift was however accepted.

\section{Discussion}

While continental adjustment could not be perfectly achieved in a globe of unaltered dimension, some observers pointed that detached continents can be appropriately adjusted in a globe of reduced dimension. I. O. Yarkovskii ${ }^{5}$ of Russia for the first-time suggested Earth's expansion in 1888 while after that many scientists, some of them independently, conceived the idea of expansion of the Earth. During the last century S.W. Carey ${ }^{6,7,8}$ of Australia relentlessly attempted to revive and establish the concept of expansion of Earth for more than five decades. O.C. Hilgenberg $^{9}$ of Germany in 1933 showed that if the globe could be reduced to two-third of its radial thickness, all the continents would be appropriately fitted.

It may be pointed out that as the mantle is solid and rigid, continental movement or drift would not be possible while expansion of the planet in that case would be well neigh impossible. Here it has been reasoned out that during the past, condition of the Mantle was congenial for planetary expansion. In this article based on Hilgenberg's model of Earth expansion ${ }^{9}$, an attempt has been made to understand the total Earth system $\left(\operatorname{Sen}^{10-17}\right)$.

\section{Mantle Condition of the Original Small Earth}

We have shown here that during the Earth's pre-expansion stage, when the planet was small and devoid of oceans, condition of the Mantle must have been semi-fluid. This is because at that stage the planet's ocean-forming water was associated with the Mantle, thereby, turning that geosphere sufficiently fluid and suitable for expansion. This crucial interpretation has been derived based on the results of experimental studies conducted by Roy and Tuttle ${ }^{-18}$ which confirmed depression of melting point of silicate rocks containing large amount of water under pressure.

\section{Influence of an Approaching Extra-Terrestrial Object on Earth}

Based Roy and Tuttle's work, as discussed in the previous section, we can conclude that only in case of semi-fluid condition of the Mantle, expansion of Earth or continental drift could be conceivable. This could be implemented by gravitational attraction of an extra-terrestrial planetary object. Initially such an object must have been absent from the vicinity of the Earth, otherwise the planetary expansion would have taken earlier.

Magnetic Influence on Earth by an Approaching Planetary Body

Dott, and Batten ${ }^{19}$ with the help of several maps of North America have shown gradual deflection of the Equator from Late Cambrian (about 480 million years ago) - when it was 
situated almost at right angle to its present position to Early Cenozoic (about 50 to 60 million years ago), when the Equator became almost parallel to its present alignment. This remarkable change in the arrangement of Equator, causing complete change in climatic condition of the Earth was possible owing to approach of a smaller planetary body with magnetic property towards the Earth. It has been considered here that the smaller planet magnetically influenced the larger planet Earth, due to which the latter experienced a smooth turn or deflection in space. In consequence, over the Earth's surface drastic changes of climatic zones took place, such as the hot and desertic area became cold or polar zone and polar zone turned into hot area.

\section{Effect of Gravitational Attraction of Moon on Earth}

In due course the smaller planetary object was captured by the larger planet Earth and became its solitary satellite, the Moon. At that stage Earth's Mantle was sufficiently fluid owing to incorporation of ocean-forming water, as discussed earlier. Since capture, the Moon started to revolve around the Earth and, in doing so, gravitationally attracted the larger planet causing its semi-fluid Mantle to bulge, thereby triggering expansion of the planet. In consequence of expansion several long sinuous cracks over the Crust were developed through which profuse amounts of basaltic magma expelled out spreading on both sides of the expansion cracks. As the process of expansion steadily progressed associated with emission of lava through the expansion cracks that turned out to be mid-oceanic ridges, the space on both sides of the crack was continuously filled up with basaltic magma, thereby, forming rudimentary ocean basins. With further expansion, the dimension of the oceans was further increased, eventually developing as large oceans. All these apparently distinct processes occurred concurrently and continued till the Mantle remained as semifluid. Along with lava emission large amounts of volatiles, chiefly composed of water vapour, also came out which on condensation deposited over the ocean basins as ocean water. However, when the planet was sufficiently expanded, due to desiccation the Mantle became solid when the process of expansion, as well as enlargement of oceans and all associated processes were stopped.

\section{Effect of Gravitational Attraction of Moon on Earth's Interior}

The effect of planetary expansion was more pronounced in the interior of the Earth compared to the changes brought about over the surface of the planet. In the interior below the Crust of $33 \mathrm{~km}$ thickness of granitic composition occurs a $2867 \mathrm{~km}$ thick geosphere of basaltic composition, known as Mantle which was originally semi-fluid due to association of ocean-forming water. Subsequently, because of loss of water this geosphere turned into a rigid and solid body. The solid Mantle is followed by the $2220 \mathrm{~km}$ thick geosphere, termed Outer Core, which has been confirmed to be fluid by seismic studies $^{20}$. although whether it is in liquid or gaseous state has not been firmly established. The prevalent view, nevertheless, considers that the fluid geosphere is mainly composed of liquid iron with some nickel, without assigning any proper reason. The inner-most $1251 \mathrm{~km}$. part of Earth is composed of solid iron with some nickel and even though magnetic lines of force emerge from this geosphere, the prevalent view, in consideration of its high temperature condition, supposed to be of the order of $350^{\circ}$ to $400^{\circ}$ Celsius, do not ascribe magnetic property of the planet to this geosphere.

\section{Continental Drift Vis-à-Vis Earth's Expansion in the Light of Opening up of Outer Core}

A large number of geologists believe that the Earth's dimension has remained the same throughout the past geological ages. If we try to adjust the continental fragments in such a globe, perfect fitting would not be possible. It may be noted that many observers have tried to adjust continents, considering unaltered dimension of the globe throughout the past geological ages. Nevertheless, as discussed earlier, in all such attempts, including the one done by Alfred Wegener ${ }^{2}$, the shores of the continents had to be distorted which show that this is not the correct way of solving problems related adjustment of continents. It has been shown by O.C. Hilgenberg ${ }^{9}$ that in a model of Earth reduced to two-third of its radial thickness perfect fitting of continents could be achieved.

\section{Earth's Expansion and Opening up of a Void Zone in the Earth's Interior}

The figure of two-third mentioned by Hilgenberg is not exact but expected to be close to two-third. Earth's total thickness being 6371 a reduction of the order of $2124 \mathrm{~km}$ is required which fairly matches with the thickness of the Outer Core which is $2220 \mathrm{~km}$.

This reasoning throws a vital clue that the so-called Outer Core has been opened up owing to planetary expansion. Here we can further interpret that before expansion of the small Earth, the Outer Core was not developed and, therefore, at that stage the Inner Core and Mantle were juxtaposed to each other. It was owing to expansion of the planet, caused by the Moon's gravitational attraction, the Mantle-Inner Core conjunction was ruptured and along the ruptured surface the so-called Outer Core was gradually opened up as a void zone. Owing to influx of particles and volatiles from the adjoining mantle, the newly opened up Outer Core behaved like a fluid-like zone, though principally retaining its void characteristics. Instead of calling this geosphere as the fluid Outer Core we prefer to call this apparently enigmatic zone as Psedo Fluid geosphere.

\section{Occurrence of Reversely Directed Force of Gravitation in Inner Core}

From the discussion made in the previous section it can be conceived that two solid geospheres Mantle and Inner Core are separated by the newly opened up virtually void zone termed, Pseudo Fluid geosphere, also known as Ourter Core according to the prevalent concept. Here we infer that such a disposition of geospheres in the interior of the planet, namely two solid geospheres of Mantle and Inner Core separated by a virtually void Pseudo Fluid geosphere would give rise to a force of gravitation in reverse or upward direction, in addition to the normal inwardly directed force of gravitation of the planet. Owing to occurrence such force of reverse gravity in the deep interior of the Earth, temperature and pressure condition of Inner Core would be sufficiently low. Therefore we can reasonably deduce that 
the Earth's Inner Core is a magnet - a view strongly supported by emergence of magnetic lines of force from that geosphere.

\section{Magnetic Influence of a Planetary Body on Earth}

As discussed previously, when the planet was not expanded and the Pseudo fluid geosphere which is a virtually void zone was not opened up, the Mantle and Inner Core were juxtaposed to each other. At that stage due to magnetic influence of an external planetary body, the Earth experienced a $90^{\circ}$ twist or rotation causing major changes in climatic characteristics of the planet. However, in later stage after opening up of the Pseudo Fluid geosphere (Outer Core), for some time the Inner Core was attached with the Mantle and consequently due to magnetic influence of an extra-terrestrial planetary object the Earth experienced a shift of $90^{\circ}$ Degree. However, after complete opening up of a void Pseudo Fluid geosphere, when the Inner Core was fully encased within the former with adequate space inside, the planet started to exhibit new magnetic phenomena like magnetic pole reversal, magnetic polar wandering etc. (Figure 3). These can be readily explained to have taken place due to magnetic influence of an external planetary body on Earth.

\section{The Planetary Expansion Occurred Uniformly}

With respect to the entire terrestrial body planetary expansion took place throughout in a uniform manner without causing protrusion in certain parts of the planet. This is because as the Moon was revolving around the Earth, its influence was equally distributed throughout the planet. At the same time the Earth was revolving around its own axis of rotation as a result of which Moon's influence was evenly distributed throughout the planet.

\section{Cause of Mountain Formation}

It has been considered that mountain like structure is formed in places where one continental block collides with another similar block while in case of expansion, the fragmented blocks move away from one another. Consequently, in case of Earth's expansion formation of mountain would not be possible. It may be noted that there is a formidable force always associated with the planet caused by rotation of the planet around its axis of rotation. This force which is maximum near the Equator and minimum near the Polar regions, can push one landmass on another and bring forth major changes over the Earth's surface, including formation of mountain ranges.

\section{Conclusion}

Here we have shown that it was principally owing to gravitational attraction of an approaching extra-terrestrial planetary body, which in due course was captured by the Earth, several major changes initially related to climatic aspect of the planet took place. The captured smaller planet eventually turned into the Earth's only satellite, the Moon, which in course of its revolution around the former, by dint of its gravitational pull, caused massive expansion of the bigger planet Earth. Such enormous expansion of nearly one/third of the planet's radial thickness which was executed in a steady manner, triggered several major changes over the
Earth's Crust including formation of mid-oceanic ridges and ocean bodies, segregation of continents etc. In the interior of the planet too, because of the Moon's gravitational attraction major changes such as opening up of a void-like zone, termed Pseudo Fluid geosphere took place. At depth such arrangement of occurrence of two solid geospheres sandwiched between a void Pseudo Fluid geosphere would generate a force of gravitation in reverse direction, in addition to the normal force of gravitation. Because of reverse gravity, sufficiently low temperature and pressure condition at deep interior of the planet prevailed supporting magnetic characteristics of the Inner Core. Before expansion when Inner Core and Mantle were juxtaposed to each other, due to magnetic influence of an approaching planetary body, Earth's disposition in space was changed, bringing forth major alteration in climatic condition of the planet. When the Pseudo Fluid geosphere was fully developed, the Earth's magnetic Inner Core was capable of executing smooth rotation within the planet owing to magnetic action of an extra-terrestrial magnetic object, giving rise to new magnetic phenomena, like magnetic pole reversal and polar wandering.

\section{References}

[1] Ortelius Abraham. Thesaurus Geographicus (in Latin), 3rd Edition, Antwerp Plantin. OCLC 214324616; 1596.

[2] Wegener Alfred. Die Entstehung der kontinente, Petermann Mitteilungn. 1912; 185-195,253-256,305309.

[3] Snider-Pellegrini Antonio. La Creation et ses Mysters Devoiles, Librarie A. Franck \& Dentu, Paris; 1858.

[4] Jeffreys Harold, 1926, The Earth, its Origin, History and Physical Constitution, $4^{\text {th }}$ Edition, Cambridge University Press, Cambridge)

[5] Yarkovsky, Ivan Osipovich, 1888, Hypothese cinetique de la Gravitation universelle et connexion avec la formation des elements chimiques, 134p, Moskau

[6] Carey, S. W. 1958, The tectonic approach to continental drift, Continental Drift - A Symposium, University of Tasmania, Hobart: p 177-355

[7] Carey, S. W., 1976, The Expanding Earth, Elsevier, Amsterdam, 488 p.

[8] Carey, S. W., 1988, Theories of the Earth and universe: a history of dogma in the earth sciences, Stanford University Press, ISBN 0-8047-1364-2

[9] Hilgenberg O. C. Vom Wachsenden Erdball, Berlin: Giessmann \& Bartsch; 1933.

[10] Sen, Subhasis, 1984, Unified global tectonics - a new qualitative approach in Earth sciences, Indian Science Congress, Geology and Geography Section, Ranchi, January 3-8, 71st Session, Abstract, p.23-24, Also in Jour. Min. Met. \& Fuels, v.32, p.20-22.

[11]Sen, Subhasis,2003 a, The nature of the Earth's inner core - what the meteorites tend to tell us, Indian Science Congress, Earth System Sciences, Bangalore, January 3-8, 2003, 90th Session, Abstract, p.55-57, Also in Jour. of Geol. Soc. India, v.61, p.364

[12] Sen, Subhasis, 2003 b, Unified global tectonics: structure and dynamics of the total Earth system, Indian Science Congress, Earth System Sciences, Bangalore, January 3-8, 2003, 90th Session, Abstract, p.57-58, Also in Jour. Min. Met. \& Fuels, v.51, p.351-355. 
[13] Sen. Subhasis, 2007, Earth -The Planet Extraordinary, Allied Publishers, New Delhi, 232p, New Delhi, India

[14] Sen, Subhasis, 2013, Surface Structure of Planetary Bodies - The Moon., Everyman's Science, vol XLVIII, no. 4 , Oct-Nov, p. 305-308

[15] Sen, Subhasis, 2015, Earth Science Revisited: Expansion-Based Hypothesis for Explaining Global Phenomena, Jour. Geol. Geophy, vol 4. Issue 6. p 4-6, 227

[16] Sen, Subhasis, 2016, Continental Drift and Plate Tectonics Vis-à-Vis Earth's Expansion: Probing the Missing Link in Understanding the Total Earth System, Jour Geol. Geophy, 5- 263, vol. 5. Issue 6

[17] Sen, Subhasis, 2021, Expansion-Oriented View on Origin of Oceans, Jour. Marine Scie Res Ocean, vol 54, issue 1, p 181-182.

[18] Roy, R. and Tuttle, O.F., 1961, Investigation under hydrothermal conditions, In Ahrens, I.H., Rankama, A.K. and Runcorn, S.K. (Eds.), Physics and Chemistry of the Earth, v.1,p.138-180, Pergamon Press, New York.

[19]Dott, Jr. Robert H and Batten, Roger , L, 1976 Evolution of the Earth, International Student Edition, $2^{\text {nd }}$. Ed. McGraw-Hill, Inc, London

[20]Bullen K.E.1965. An Introduction to the theory of Seismology, Cambridge University Press, London

\section{Figures}

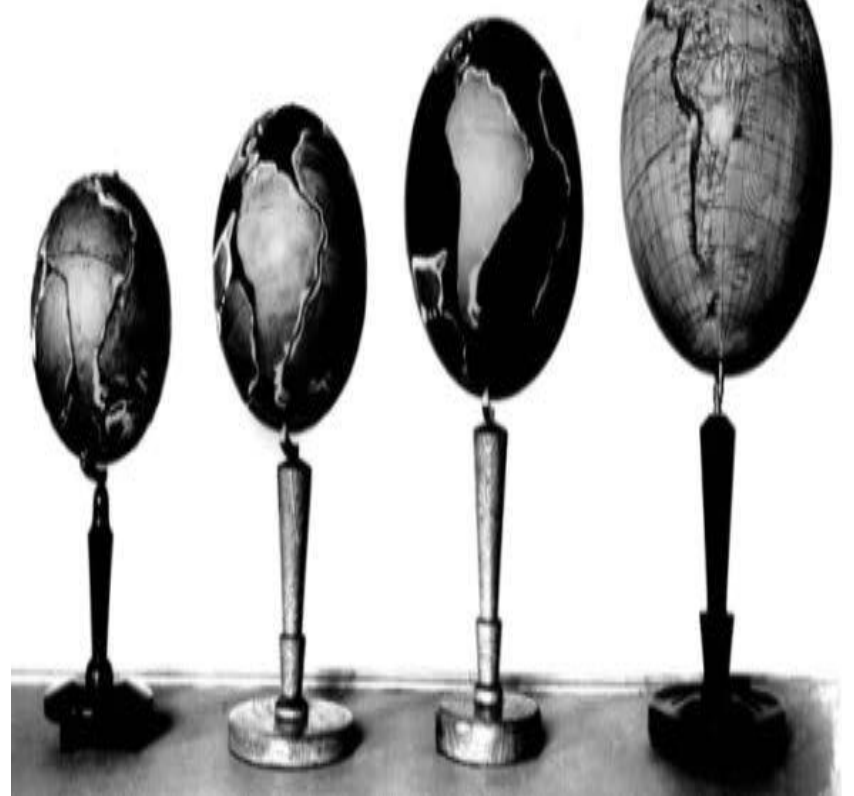

Figure 1: O.C. Hilgenberg of Germany in 1933 showed that if the radius in a model of Earth could be reduced to twothird of its radial thickness, all the continental blocks could be adjusted in a perfect manner. It can be noted that in the primordial small Earth, there were no oceans although lakes were present. Hence, it can be conceived that at that time the ocean forming water was associated with the Mantle turning it considerably fluid and predominantly suitable for expansion (Sen, 1984-2007).

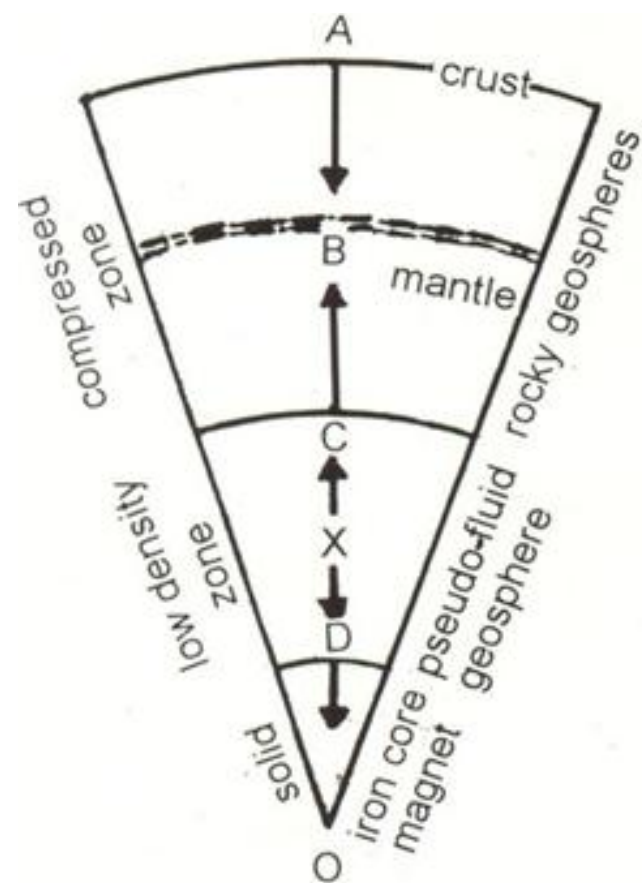

Figure 2: Overall structure of the Earth (not to scale) showing trend of gravitational attraction on surface and in the interior of the planet. The Mantle and Inner Core (iron core) are separated by a virtually void geosphere, shown here as Pseudo Fluid geosphere of low density as through this zone seismic wave passes, indicating that it may not be completely void zone. At the deep interior of the Earth, covering the Inner Core, in addition to normal down-ward direction of gravitation, an additional force of gravitation in reverse direction would occur, Because of prevalence of reverse gravity, the temperature-pressure condition at depth would be low and Inner Core is therefore a magnet.

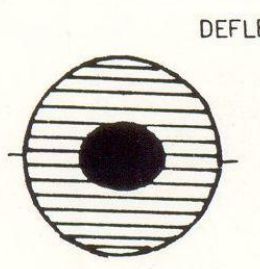

(a)

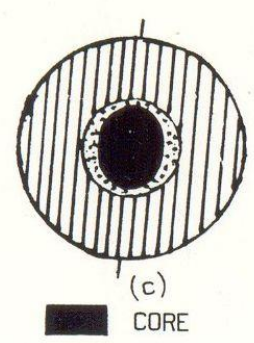

DIII MANTLE
DEFLECTION OF THE EARTH

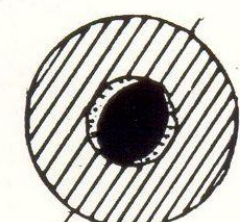

(b)

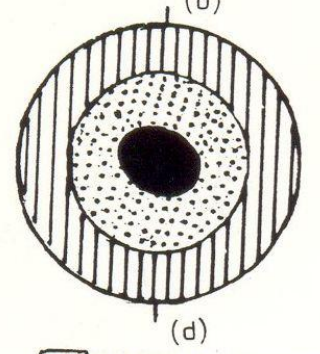

渴PSEUOO-FLUID ZONE

(not to scale)
Figure- 3, Before expansion of the planet, the solid iron core and semi-fluid Mantle were juxtaposed to each other (Fig. a). Due to expansion the original Core-Mantle conjunction was ruptured (Fig. b) and along the ruptured surface a void zone or Pseudo-Fluid geosphere was developed (Fig .c). Hence, due to occurrence of two solid geospheres separated by a virtually void zone, in the deep interior, the phenomenon of reverse gravity would prevail, generating low temperature and low-pressure zones in the Earth's Core 
and deeper parts. After development of the thick PseudoFluid geosphere (Fig. d), the iron-core remained within it in a suspended condition. Hence at this stage in response to extra-terrestrial magnetic influences, polar wandering, pole reversal and west-ward drift could take place which have been precisely documented over the global surface (not to scale). 